\title{
Application on the Neural Network Information Fusion Technology
}

\author{
Zhu Sen ${ }^{1, a}$, Bai Xuelian ${ }^{2, a}$, Chen Hongmin ${ }^{3, b}$ and Zhang Minghu ${ }^{2, b}$ \\ ${ }^{1}$ Simulation Training Center, Dalian Naval Academy, Dalian, China \\ ${ }^{2}$ Dept. of Underwater Weaponry and Chemical Defense, Dalian Naval Academy, Dalian, China \\ ${ }^{3}$ The Detachment of Warship Training, Dalian Naval Academy, Dalian, China

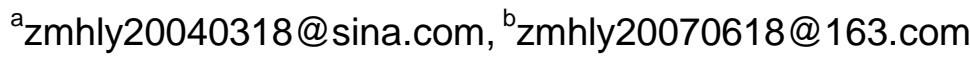

Keywords: Wavelet Neural Network, model design thought, feature extraction, pattern recognition.

\begin{abstract}
Aims at the characteristics of the chemical agents signals, the pattern recognition method based on the wavelet neural network(WNN) is put forward. Firstly, the model design thought of the WNN pattern recognition technology for chemical agents are analyzed, which shows that the wavelet analysis has good time-frequency features due to its capability of localizing and differentiating the high and low frequencies of a signal and keeping the time domain features of the original signal, as a result, the wavelet transform can effectively extract the feature of the chemical agents signals; Secondly, the model and learning algorithm for the WNN are constructed, which implements that the pattern recognition method for the chemical agents combines with the advantage of the wavelet and $\mathrm{NN}$, the wavelet transform method as a fore processing medium is used to extract the feature which reflects the information of the chemical agents, and the features are fed into the $\mathrm{NN}$ as the input patterns for training and classifying, to achieve the intelligence distinguishing; Lastly, the results of the examples and simulated test show that: this method is workable, and of the high identification accuracy, remarkable generalization capability, good stability, and high speed and high reliability.
\end{abstract}

\section{Introduction}

The wavelet transform is a kind of the signal time-frequency analysis methods, of the characteristics of multi-recognition-ratios analysis, and furthermore, of the ability of giving expression to local features at both time and frequency domains. The wavelet transform is of the self-adaptability of the signal, and it is very fit for detecting momentary abnormal phenomenon hidden in the normal signal and displaying its components. Using the continuous wavelet transform to process identification and detection for the dynamic system of chemical agent is of nicer results.

For implementing effectively detection and rapid exact identification for the chemical agents in sea-battlefield, this paper studies the application about pattern recognition(PR) technology in Naval Ships Chemical Detection (NSCD) field. The PR model is built. At the same time, connecting the wavelet analysis with the neural networks(NN) organically, and based on the wavelet transfer and the $\mathrm{NN}$, the model of the speedy features extraction and identification for chemical agents is founded.

\section{Model design thought}

Generally speaking, there are three steps in the PR of pattern collection, feature extraction or selection and class judgment. After the pattern collection and pretreatment, the pattern space is formed. The dimensions are reduced by the feature extraction or selection, and transformed the pattern space into the characteristic space. And the type space is obtained by the class judgment of the classifier. Therefore, the structures of the PR system show that to establish the three spaces and their recursive transformation.

The mode samples, in fact, is the various training samples of this PR system; the samples form the pattern space. In order to make the every pattern sample contained the useful information of the chemical agents: Firstly, the gathered samples must be pretreated with the wavelet package analysis technology, one of the modern applied analysis technologies, to extract the feature information, and 
the transformation from the pattern space to the characteristic space were realized; Secondly, the transformation form the characteristic space to the type space adopted the traditional PR method as well as imported the PR means of the NN, which the processes of the pattern space transformation, and the characteristic space and the type space recursive transformation fuse into one; Lastly, using the PR model recognized the various samples, and drew a series of the recognition conclusions and analyzed briefly the recognition conclusions. The basic thought of realizing the PR model for chemical agents is the data pretreatment (constructing pattern space), feature information extraction or selection of the chemical agents (constructing characteristic space), classifiers design (constructing type space), and recognition conclusions analysis (material application).

\section{Wavelet feature extraction}

In the process of distinguishing the chemical agents, the practical data of many parameters can be gotten by chemical sensors. These data contain rich information of the chemical agents. Feature extraction or selection is the powerful tool to reduce the mode dimension. Reducing dimension of the primitive features can help minimize the wrong recognition rate of the classified device. Via wavelet package analysis technology, the signals energy data of various frequencies can be recorded, thus the frequency energy feature was gotten, which distinguishes one from another. Choosing feature parameter is based on choosing the sample data's features. With parameter features' comprehensive discrete degree or recognition credit degree as a criterion, a group of better feature parameters to recognize can be gotten.

The basic means and steps to analyze the chemical agent energy spectrum with wavelet analysis are as follows:

(1) Firstly, calculating the residual signal of a certain feature parameter: the residual signal $=$ the chemical agent signal - the normal signal

(2) Secondly, analyzing the residual signal with the wavelet analysis of three levels, and distilling the third level's 8 signal features ranging from low frequency band to high frequency band.

(3) Thirdly, reconstructing the wavelet analysis coefficient, and distilling the signals of every frequency zone. Using the reorganized signal- $S_{30}$ to stand for $X_{30}, S_{31}$ to stand for $X_{31}$, and so on. Here, only analyzing all the nodes on the third level. Thus the formula of the overall signal $\mathrm{S}$ is as follows:

$$
\mathrm{S}=\mathrm{S}_{30}+\mathrm{S}_{31}+\mathrm{S}_{32}+\mathrm{S}_{33}+\mathrm{S}_{34}+\mathrm{S}_{35}+\mathrm{S}_{36}+\mathrm{S}_{37} \text {. }
$$

(4) Fourthly, obtaining the whole energy of every frequency signal, the input signal is a random signal, so the output is also a random signal. Supposing the corresponding energy of the $S_{3 j}(j=0,1$, $2, \ldots, 7)$ is $E_{3 j}(j=0,1,2, \ldots, 7)$, the formula is as follows:

$$
E_{3 j} \int\left|S_{3 j}(t)\right|^{2} d t=\sum_{k=1}^{n}\left|x_{j k}\right|^{2} \text {. }
$$

In the formula: $x_{j k}(j=0,1, \ldots, 7 ; \quad k=1,2, \ldots, n)$ stand for the discrete amplitude of the reorganized signal $\mathrm{S}_{3 \mathrm{j}}$.

(5) Lastly, structuring eigenvector. When the chemical agent emerges in the system, it brings the bigger effect for the energy of every frequency band signal. So used the energy as the element, the eigenvector can be structured, the eigenvector $\mathrm{T}$ is as follows:

$$
T=\left|\begin{array}{llllllll}
E_{30} & E_{31} & E_{32} & E_{33} & E_{34} & E_{35} & E_{36} & E_{37}
\end{array}\right| .
$$

When the energy is bigger, usually, $E_{3 j}(j=0,1,2, \ldots, 7)$ is a bigger numerical value, which shall bring some inconvenience form the data analyses. Consequently, the eigenvector $\mathrm{T}$ can be reformed, that is to say, treated by normalization. The new vector form is as follows:

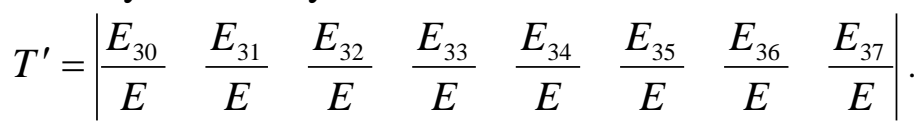

In the formula: 


$$
E=\left(\sum_{j=0}^{7}\left|E_{3 j}\right|^{2}\right)^{1 / 2} .
$$

The new vector $T^{\prime}$ is the normalized vector.

The experimental data from chemical sensors require that the input signals are of the rich frequency elements, which can cover the various frequencies range of the system from low frequency to high frequency. With the above mentioned way, the feature vectors can be structured based on the signals both in the normal situation and with the chemical agents. According to the basic way of wavelet analysis of energy spectrum, the output vectors of the normal signal, chemical agents' signal and residual signal are worked out respectively, from which, that the different chemical agents obtain the different frequency energy feature vectors are known. They reflect the different chemical agents, and which provides the practical feature vectors for the recognition of chemical agents based on the NN. According to the feature vector to work out the sort decision-making, i.e., to confirm the chemical agent that it is which kind of chemical agents.

\section{WNN model}

The wavelet NN (WNN) is a kind of the multilayer forward propagation network based on the wavelet analysis, which can avoid the local optimization for the network training, quicken the constringency speed, and bear the strong learning and remarkable generalization capability.

The difference between the WNN and forward propagation network is that the activation function isn't the Sigmoid non-linearity function but the wavelet group function. For one multi-input and multi-output mapping $f: R^{m} \rightarrow R^{q}$, its network model may express:

$$
\hat{y}_{i}(t)=\hat{f}_{i}(t)=\phi\left[\sum_{j=1}^{p} w_{i j}\left(\sum_{k=1}^{m} x_{k}(t) \psi\left(\frac{k-b_{j}}{a_{j}}\right)\right)\right] .
$$

In the formula: $i=1,2, \ldots, q ; x_{k}(t)(k=1,2, \ldots, m)$ is the No. $k$ input variable; $\hat{y}_{i}(t)(i=1,2, \ldots, q)$ is the No. $i$ output variable; $p$ is the number of the latent layer cell; the weight function, from the input layer No. $k$ cell to the latent layer No. $j$ cell, is the wavelet function $\psi\left(\frac{k-b_{j}}{a_{j}}\right), a_{j}, b_{j}$ serve respectively as the scale and displacement of the wavelet function; $w_{i j}$ is the join weight from the latent layer No. $i$ cell to the output layer No. $j$ cell; $\phi(\bullet)$ is the Sigmoid non-linearity function. The whole network parameter trained are $w_{i j}, a_{j}$ and $b_{j}$, which they can be obtained by the again and again learning.

\section{Experiment and simulation}

To verify the validity of the model, using the multi-sensors fusion system, the response data of six different chemical agents, like GB, GD, VX, HD, L, AC, are obtained by experiment under normal temperature condition with the multi-sensors array. In experiment, the concentration of neural agent ranges from $0.01 \mathrm{mg} / \mathrm{m}^{3}$ to $100 \mathrm{mg} / \mathrm{m}^{3}$, vesicant agent ranges from $1.0 \mathrm{mg} / \mathrm{m}^{3}$ to $100 \mathrm{mg} / \mathrm{m}^{3}$, and AC from $10 \mathrm{mg} / \mathrm{m}^{3}$ to $1000 \mathrm{mg} / \mathrm{m}^{3}$; based on the simulation natural environment of the sea battlefield, the experiment is divided into the following four cases: the single chemical agent without interference, single chemical agent with interference, mixed chemical agents without interference, and mixed chemical agents with inference.

Firstly, the wavelet package analysis technology is executed for the observed data of the single chemical agent without interference, the energy of the chemical agent's signals at various frequency zones is counted, and a kind of the frequency zone energy feature using recognition is obtained, in which a group of better feature parameters is selected to recognize the chemical agent, using the comprehensive discrete degree or recognition confidence-degree of features parameter samples as a 
criterion; Secondly, the wavelet package analysis technology is executed for the observed data of other three cases, and the feature parameters of the chemical agents are extracted to distinguish the chemical agents efficiently; Lastly, on the basis of obtaining experiment data to a certain extent, the simulation experiment is set up; in the processes of experimentation and simulation, the mass correlative data of the chemical agent recognition system can be obtained, and these data reflected directly or indirectly the rich information of the chemical agent form different aspects; based on the extraction or selection of the features information and data processing relatively for the chemical agent, the chemical agents can be distinguished by the model system.

\section{Conclusion}

Using the WNN PR technology can realize the intelligent distinguishing for the chemical agents. Based on the experimental research, through the experiment and simulation, using the wavelet signal for the chemical agents to extract or select the features, and the conclusions show: Different agents get the different feature vectors of frequency band energy by wavelet analysis technology, the corresponding wavelet energy distribution eigenvector of the parameter residual data can be obtained by treating the chemical agent sample residual data, which can construct the learning sample of the recognition NN. The chemical agents can be confirmed by the feature vectors classified decision-making, and the results are of repeatability. It is completely feasible that the analyses for the chemical agents with the WNN technology.

\section{Acknowledgements}

This work was financially supported in part by the National Defense Pre-Research Found of China (41101050403).

\section{References}

[1] Y. Zhu, E. Song, J. Zhou, et al. IEEE Transactions on Signal Processing, No. 5(2005), p. 1631.

[2] L. Wang, L. Zhang, J. Kang. Transactions of Beijing Institute of Tech., No.2 (2006), p. 120.

[3] M. Zhang, G. Pang, H. Liu, et al. Journal of Guangxi Normal University: Natural Science Edition, No.1 (2008), p. 220.

[4] X. Liu. Journal of Liaoning Shihua University, No. 3(2010), p. 79.

[5] H. Zhou. Journal of Chifeng University (Natural Science Edition), No. 5(2011), p. 96.

[6] L. Qiu, T. Liu. Journal of Minjiang University, No. 2(2013), p. 88.

[7] Y. Ao, B. Wang. Machine Tool \& Hydraulics, No. 13(2014), p. 168.

[8] J. Liu, P. Wang, L. Yang. Journal of Beijing University of Technology, No. 6(2014), p. 843.

[9] X. Tian, Zh. Ma, Sh. Tan. Journal of Sichuan Ordnance, No. 5(2015), p. 90. 